\title{
Epoxy, ZnO, and PTFE nanocomposite: friction and wear optimization
}

\author{
N.L. McCook, B. Boesl, D.L. Burris and W.G. Sawyer* \\ Department of Mechanical and Aerospace Engineering, University of Florida, Gainesville, FL , 32611, USA
}

Received 6 February 2006; accepted 9 June 2006

\begin{abstract}
To lower the friction coefficient and increase the wear resistance of epoxy, nanoparticles of zinc oxide and polytetrafluoroethylene (PTFE) were added in small volume percents to an epoxy matrix. Tribological testing of the samples in this study was completed on a linear reciprocating tribometer with a $250 \mathrm{~N}$ normal load and a $50.8 \mathrm{~mm} / \mathrm{s}$ sliding speed. Several samples were made and tested following a modified Simplex Method optimization procedure in order to find a volume percent for optimized wear resistance and friction coefficient. The sample with the optimum wear rate consisted of 1 volume percent of zinc oxide nanoparticles and 14.5 volume percent of PTFE nanoparticles. It had a wear rate of $k=1.79 \times 10^{-} 7 \mathrm{~mm}^{3} / \mathrm{Nm} ; 400 \times$ more wear resistant than neat epoxy. The sample with the optimum friction coefficient consisted of 3.5 volume percent of zinc oxide nanoparticles and 14.5 volume percent of PTFE nanoparticles and had a friction coefficient of $\mu=0.113$, which is almost a $7 \times$ decrease in friction coefficient from neat epoxy.
\end{abstract}

KEY WORDS: PTFE, epoxy, zinc oxide, nanocomposites, optimization, Simplex Method, wear rate, friction coefficient

\section{Introduction}

Epoxy alone has a high friction coefficient in most applications, as well as poor wear resistance compared to epoxy containing composites. Fillers have been added to reduce both friction coefficient and wear rate. Studies with epoxy and micron-scale fillers by Burroughs et al. [1], Zhang et al. [2], and Chang et al. [3], show a monotonic reduction in wear rate with increased filler loading up to 30 volume percent $\left(\mathrm{vol}_{\%} \%\right)$. Nanoscale fillers such as, $\mathrm{TiO}_{2}[2,3], \mathrm{SiO}_{2}$ [4], $\mathrm{Al}_{2} \mathrm{O}_{3}$ [5,6], and $\mathrm{Si}_{3} \mathrm{~N}_{4}$ [7] have also been shown to reduce friction and wear in epoxy matrix composites. Chang et al. [3] used $\mathrm{TiO}_{2}$, polytetrafluoroethylene (PTFE), short carbon fiber, and graphite as fillers in an epoxy matrix, and was able to decrease the friction coefficient to $\mu=0.35$ and decrease the wear rate $10 \times$ with $10 \mathrm{vol} \% \mathrm{TiO}_{2}$. Shi et al. [5] saw a $0.4 \times$ decrease in friction coefficient, from $\mu=0.58$ to $\mu=0.35$, and $10 \times$ decrease in wear resistance with the addition of $2.0 \mathrm{vol} \%$ nanoscale $\mathrm{Al}_{2} \mathrm{O}_{3}$ to epoxy, and Wetzel et al. [6] saw a $2 \times$ decrease in wear resistance with the addition of small amounts of nano$\mathrm{Al}_{2} \mathrm{O}_{3}$. Shi et al. [7] also saw decreases in friction coefficient, from $\mu=0.7$ to $\mu=0.32$ with the addition of up to $2.25 \mathrm{vol} \%$ of nano- $-\mathrm{Si}_{3} \mathrm{~N}_{4}$.

The mechanical properties of particle filled composites are dependent upon the number and sizes of the particle defects in the matrix, and tend to be diminished with increased filler loading. Thus, it is desirable to optimize tribological properties at low particle loading.

*E-mail: wgsawyer@ufl.edu
The filler volume fraction that resulted in the lowest wear rate of the composite (optimum loading) is plotted versus average reported filler diameter for the aforementioned studies in figure 1. The optimum volume fraction is reduced with reduced particle size motivating the use of nanoparticles in this study.

The epoxy used in this study is in the liquid phase prior to curing; this allows for easy particle dispersion as well as the ability to mold large and irregularly shaped parts. Nanoscale zinc oxide $(\mathrm{ZnO})$ is thought to provide toughness and reduce wear by arresting cracks, promoting ductility, compartmentalizing damage, and reducing the effect of the third body debris. PTFE is a known solid lubricant and is used as filler in this study to form low shear strength transfer films. The interaction of these fillers is hypothesized to be synergistic, reducing traction stresses and the amount and size of third body debris which may destroy tribologically favorable transfer films.

\section{Composite preparation and optimization}

The $\mathrm{ZnO}$ used in this study was obtained from nanophase is has an average particle size of $53 \mathrm{~nm}$. The PTFE used in this study is $200 \mathrm{~nm}$ in diameter. The nanocomposites in this study consist of epoxy, zinc oxide, and PTFE. Both the zinc oxide particles and the PTFE particles are agglomerated prior to sonication and dispersion into the epoxy resin. Scanning electron microscopy of the particles of zinc oxide and PTFE are shown in figure 2. The PTFE particles are in agglomerations of around $20 \mu \mathrm{m}$ and the zinc oxide particles 


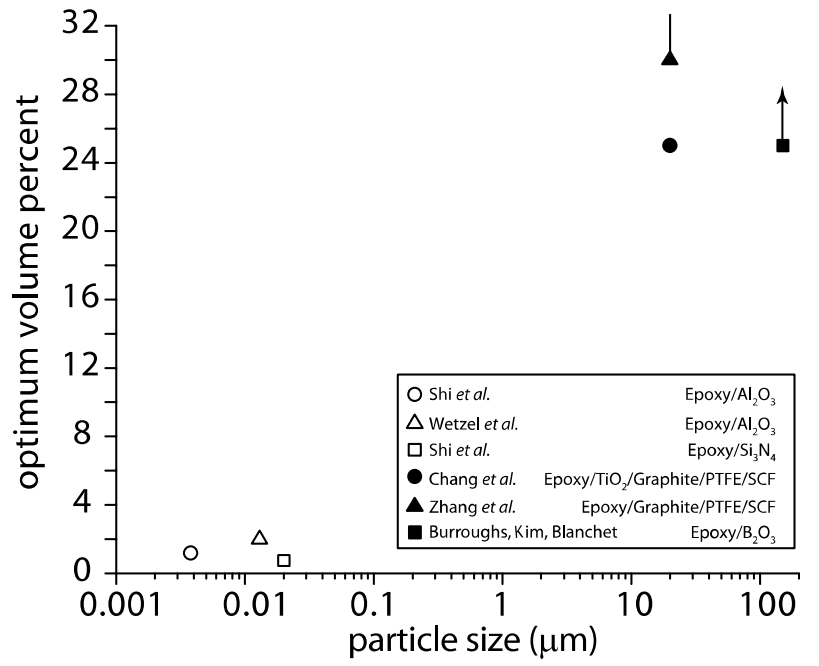

Figure 1. Plot of the volume percent correlating to optimum tribological properties according to the literature versus particle size in micrometers for various fillers in an epoxy matrix.

have some smaller agglomerations and some larger agglomerations up to $20 \mu \mathrm{m}$ in size. Similar to the process in Liao et al. [8], using acetone as a solvent, epoxy resin was mixed with a 10:2 (resin:acetone) weight percent ratio and manually stirred for $5 \mathrm{~min}$. Next, the zinc oxide and PTFE nanoparticles were mixed in slowly while stirring continuously. The mixture was then placed in a bath sonicator for $6 \mathrm{~h}$ to disperse the particles and reduce the amount and size of agglomerations of the PTFE and zinc oxide nanoparticles. Then, the mixture was placed in a vacuum oven at $75^{\circ} \mathrm{C}$ for $2 \mathrm{~h}$ to vaporize the acetone. A vacuum was slowly pulled on the oven to reduce porosity. The epoxy hardener was then added with a 10:2.5 (resin:hardener) weight percent ratio and the mixture was poured into a mold. The mixture was then cured at $60{ }^{\circ} \mathrm{C}$ for $2 \mathrm{~h}$. Once cured the specimen was removed from the mold, post-cured at $170{ }^{\circ} \mathrm{C}$ for $50 \mathrm{~min}$, and machined to the desired shape. Figure 2(b) shows a typical $\mathrm{ZnO}$ dispersion obtained from this processing.

The tribological literature is full of binary composites that have been optimized in $\mathrm{wt} \%$ or $\mathrm{vol} \%$ for a particular property or behavior. There are numerous practical examples of ternary systems that have useful tribological properties. However, optimization of these systems has not been extensively reported in the tribology literature, nor have the methodologies to perform such experimental endeavors. The purpose of this study was to attempt an optimization of a nanocomposite to obtain the lowest wear rate and friction coefficient under a prescribed set of experimental conditions. One goal of this study is to see if there is an efficient experimental process that can be used to guide the materials development without exhaustively creating and evaluating a large sample population of nanocomposites. This is useful to reduce time, costs (due to the expensive nature of nanoparticles), and increase the number of constituents. The method that we are using is widely described as a "Simplex Method" following Nelder and Mead [9], which we have modified slightly to maintain reasonable increments in constituent loadings.

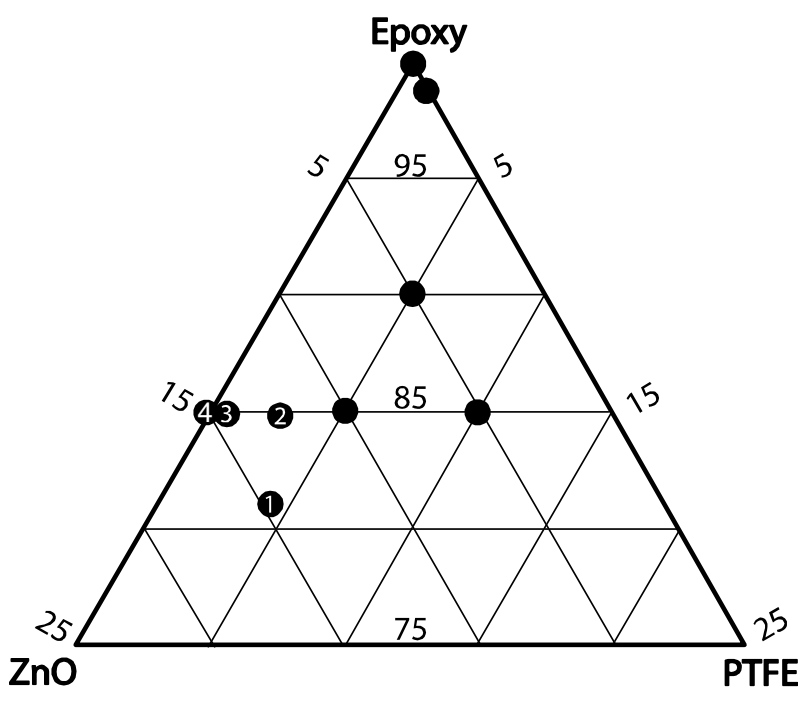

Figure 3. Ternary diagram with the matrix material, epoxy, at the pinnacle and the filler materials, $\mathrm{ZnO}$ and PTFE, at the bottom corners. The lines on the ternary diagram are line of constant volume percent. Filled circles represent tested samples and number circles represent the order of iterations. The fifth and third iteration lie on the same point indicating that the sample has been optimized.

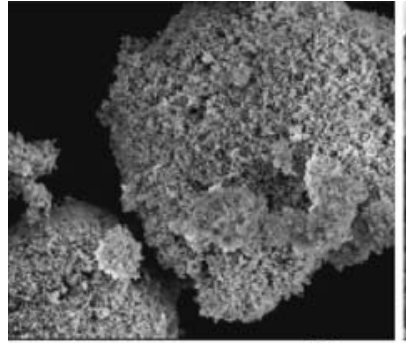

(a)

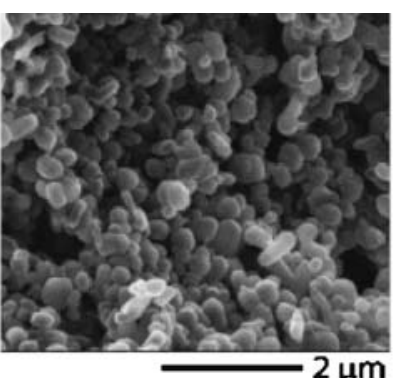

$2 \mu \mathrm{m}$

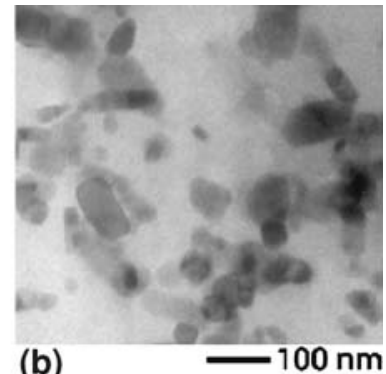

(b)

Figure 2. (a) Scanning electron microscopy of $\mathrm{ZnO}$ agglomerations and PTFE particles. (b) transmission electron microscopy of $\mathrm{ZnO}$ dispersed in an epoxy matrix. 
Table 1 .

Volume concentration of matrix material, epoxy, and filler materials, $\mathrm{ZnO}$ and PTFE along with the tabulated average values for steady state wear rate and friction coefficient.

\begin{tabular}{lcccc}
\hline \multicolumn{2}{l}{ Volume concentration } & & $k \times 10^{-6}\left(\mathrm{~mm}^{3} / \mathrm{Nm}\right)$ & $\mu$ \\
\hline Epoxy & ZnO & PTFE & & \\
\hline 85 & 5 & 10 & 0.658 & 0.163 \\
85 & 10 & 5 & 8.130 & 0.185 \\
90 & 5 & 5 & 2.290 & 0.177 \\
85 & 2.75 & 12.25 & 0.281 & 0.123 \\
82 & 3.5 & 14.5 & 0.607 & 0.113 \\
84.5 & 1 & 14.5 & 0.179 & 0.135 \\
85 & 0 & 15 & 0.370 & 0.193 \\
100 & 0 & 0 & 83.500 & 0.704 \\
99 & 1 & 0 & 6.100 & 0.273 \\
\hline
\end{tabular}

For this optimization study, three initial samples were created at processable locations on a ternary diagram. The initial three points were chosen at small volume percents of PTFE and zinc oxide nanoparticles to enable easy and robust processing. The ternary diagram shown in figure 3 represents all three components of the composite material with the matrix material, epoxy, at the top point and the fillers, zinc oxide and PTFE, on the side points. The lines on the ternary diagram are lines of constant volume percent of each constituent. Samples with less than 50 volume percent epoxy were not tested, (such high loadings inhibit epoxy curing). To find the next iteration in the search, a plane is fit to the three initial points based on the wear rates of the samples and a gradient of the plane is used to determine the search direction at a distance proportional to the gradient of the plane. Once the next point is tested, the samples' wear rate along with the lowest two wear rates from the previous samples were used to create a new plane and find the next point. This process was repeated until the optimization scheme began pointing to previously tested sample compositions. It is important to note that the optimization process used can produce a local optimum, but for this study the only processable conditions pointed repeatedly to this optimum.

\section{Experimental procedure}

Experiments for this study were conducted on a linear reciprocating pin-on-disk tribometer that has been extensively analyzed and discussed (see an uncertainty analysis of the friction coefficient and wear rate in Schmitz et al. [10,11]). The counterfaces are lapped
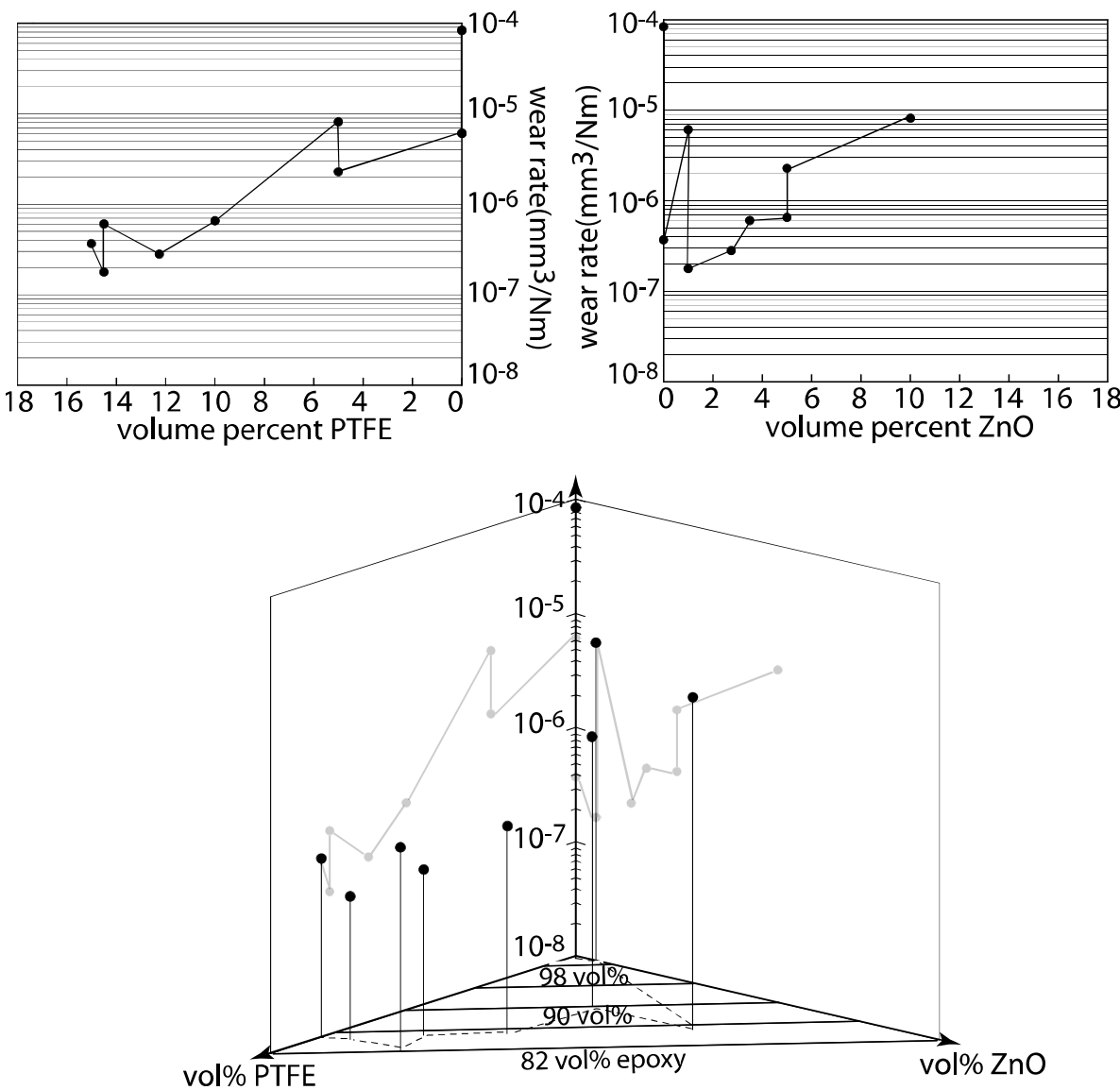

Figure 4. Steady state wear data for the epoxy nanocomposite samples in this study. These tests were run on a reciprocating tribometer under a $250 \mathrm{~N}$ load and a $50.8 \mathrm{~mm} / \mathrm{s}$ sliding speed. 
plates of AISI 304 stainless steel and are described in detail in Burris and Sawyer [12]. Five measurements on a representative sample using scanning white light interferometry resulted in $\left\langle\mathrm{R}_{\mathrm{q}}\right\rangle=161 \mathrm{~nm}$ and $\sigma=35 \mathrm{~nm}$. Composite samples are $6.35 \mathrm{~mm} \times$ $6.35 \mathrm{~mm} \times 12.7 \mathrm{~mm}$ in size and are in contact with the counterface with a normal force of $250 \mathrm{~N}(6.3 \mathrm{MPa})$. The reciprocating length is $25.4 \mathrm{~mm}$ and the average sliding speed is $50.8 \mathrm{~mm} / \mathrm{s}(1 \mathrm{~Hz})$.

Samples and counterfaces were cleaned with methanol prior to testing. Due to environmental uptake of the epoxy matrix, a gravimetric method could not be utilized to determine mass loss for samples that were very wear resistant. Thus, an LVDT was used to determine the change in height of the pin in-situ. In order to minimize the effects of creep, regressions of the wear slopes were conducted for the portion of the test determined to be at steady-state; this measurement is an upper bound on wear rate since the "worn" volume includes effects from both wear and creep. Because the wear rates of the samples vary by orders of magnitude, it was necessary to conduct tests for different distances of sliding. High wear rate materials are worn out quickly, while low wear rate samples must be run significantly longer sliding distances in order to detect statistically significant measures of wear volume.

\section{Results and discussion}

Epoxy is not inherently lubricious, with a coefficient of friction typically above $\mu=0.5$. Epoxy is frequently used as a matrix for composites because of its strength and easy processing. For this study, the epoxy matrix was originally filled with small volume percents of zinc oxide to increase the wear resistance; the wear resistance increased by more than $10 \times$ with the addition of $1 \mathrm{vol} \%$ of zinc oxide nanoparticles (see table 1). The sample with the lowest friction coefficient contained $3.5 \mathrm{vol} \%$ zinc oxide and 14.5 vol\% PTFE. Figures 4 and 5 and table 1 give all of the samples created for the study and the corresponding wear rates and friction coefficient versus the vol\% of each filler. The sample having the lowest wear rate, $k=1.79 \times 10^{-7} \mathrm{~mm}^{3} / \mathrm{Nm}$, contained $1 \mathrm{vol} \%$ zinc oxide and 14.5 vol $\%$ PTFE.

The trend for wear rate pointed to having a composite with no zinc oxide filler and $15 \mathrm{vol} \%$ PTFE, but after testing a composite with no zinc oxide and $15 \mathrm{vol} \%$
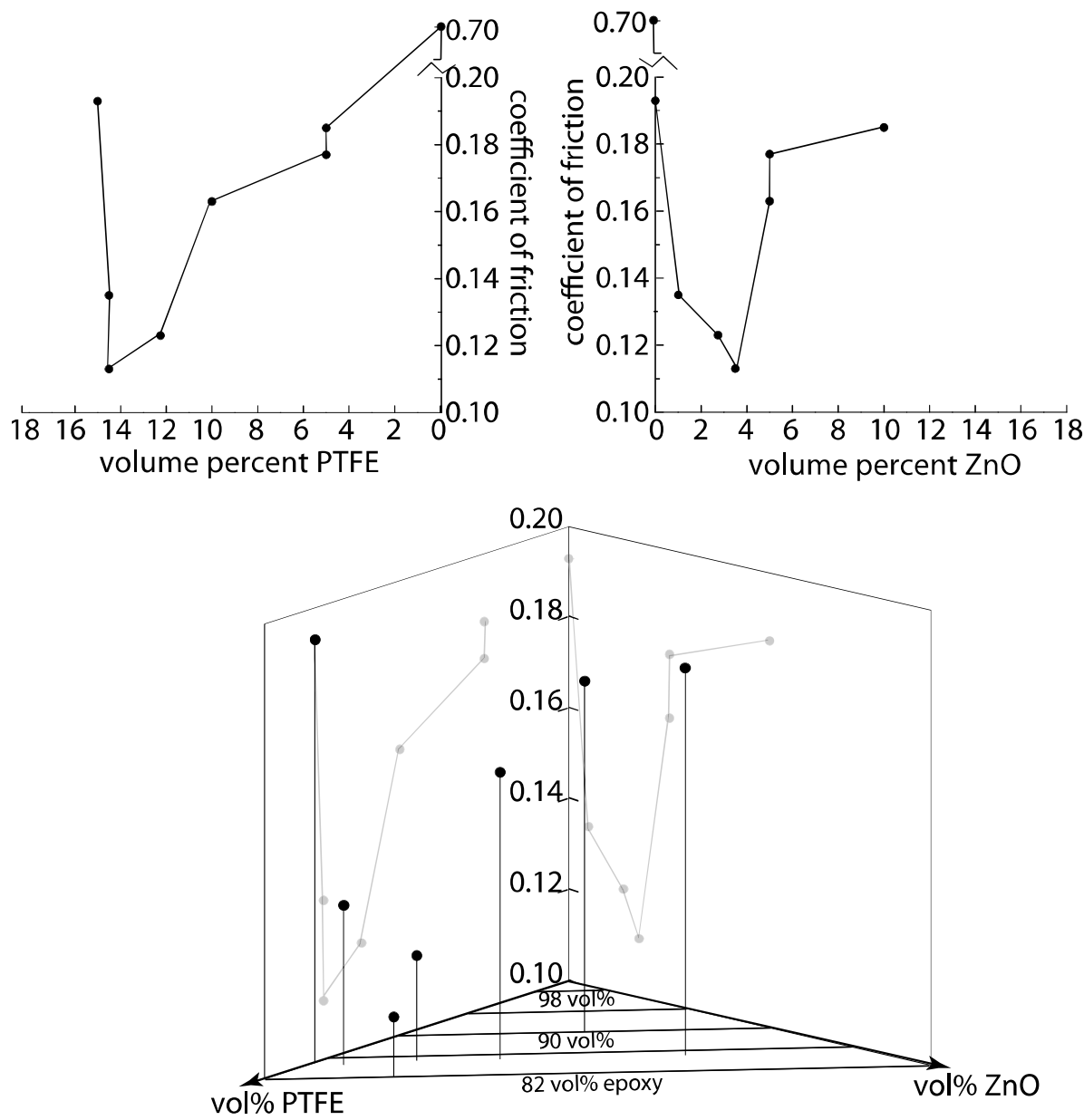

Figure 5. Average steady state friction coefficient data for the epoxy nanocomposite samples in this study. These tests were run on a reciprocating tribometer under a $250 \mathrm{~N}$ load and a $50.8 \mathrm{~mm} / \mathrm{s}$ sliding speed. 
PTFE it was proven that the addition of small amounts of zinc oxide nanoparticles were needed to obtain the lowest wear rate and friction coefficient. The wear rate and friction coefficient of the sample without zinc oxide and 15 vol $\%$ PTFE was $k=3.70 \times 10^{-7} \mathrm{~mm}^{3} / \mathrm{Nm}$ and $\mu=0.193$, respectively. Both of these values are higher than the values for the optimum samples' wear rates and coefficient of friction.

There are a number of theories regarding the origins of wear resistance and friction coefficient reductions in polymeric nanocomposites. One constant observed in experimental tribology is that fine wear debris usually accompanies reduced wear rates, reduced friction coefficient, and uniform transfer films. The mechanics and physics underlying this debris generation is an open question. A hypothesis for the reduced friction coefficient and wear rate upon the inclusion of $\mathrm{ZnO}$ in epoxy is that the wear debris size was regulated though the control of particle dispersion (figure 2b).

\section{Conclusions}

1. An experimental process that utilized a simple optimization procedure was performed on a ternary nanocomposite. This process found and validated an optimum composition after creating under a dozen samples.

2. Wear resistance of the epoxy nanocomposites was greatly increased with the addition of small volume percents of zinc oxide nanoparticles and nanoparticles of PTFE.

3. Friction coefficient decreases with the addition of zinc oxide nanoparticles and was further decreased with the addition of PTFE nanoparticles.

\section{Acknowledgments}

This material is based upon work supported under an AFOSR-MURI grant FA9550-04-1-0367. Any opinions, findings, and conclusions or recommendations expressed in this material are those of the authors and do not necessarily reflect the views of the Air Force Office of Scientific Research.

\section{References}

[1] B. Burroughs, J. Kim and T. Blanchet, Tribol. Trans. 42 (1999) 592.

[2] Z. Zhang, C. Breidt, L. Chang, F. Haupert and K. Friedrich, Composites A 35 (2004) 1385.

[3] L. Chang, Z. Zhang, C. Breidt and K. Friedrich, Wear 258 (2005) 141.

[4] X. Xing and R. Li, Wear 256 (2004) 21.

[5] G. Shi, M. Zhang, M. Rong, B. Wetzel and K. Friedrich, Wear 256 (2004) 1072.

[6] B. Wetzel, F. Haupert and M. Zhang, Compos. Sci. Technol. 63 (2003) 2055.

[7] G. Shi, M. Zhang, M. Rong, B. Wetzel and K. Friedrich, Wear 254 (2003) 784.

[8] Y. Liao, O. Marietta-Tondin, Z. Liang, C. Zhang and B. Wang, Mater. Sci. Eng. A 385 (2004) 175.

[9] J. Nelder and R. Mead, Comput. J. 7 (1965) 308.

[10] T. Schmitz, J. Action, D. Burris, J. Ziegert and W. Sawyer, J. Tribol.Trans. ASME 126 (2004) 802.

[11] T. Schmitz, J. Action, J. Ziegert and W. Sawyer, J. Tribol. Trans. ASME 127 (2005) 673

[12] D. Burris and W. Sawyer, Tribol. Trans. 48 (2005) 147. 